\title{
Cystic tumour of the atrioventricular nodal region: report of a case successfully treated with surgery
}

\author{
J R Paniagua, J R Sadaba, L A Davidson, C M Munsch
}

\begin{abstract}
A case is reported of a 59 year old woman who presented with palpitations. Electrocardiographic studies revealed atrial fibrillation and atrioventricular block. Echocardiography and magnetic resonance imaging showed a right atrial cystic mass attached to the interatrial septum. The patient underwent surgical excision of the mass. Histopathological findings were of a cystic tumour of the atrioventricular nodal region. This is the second report of this condition diagnosed antemortem and treated successfully with surgical excision.

(Heart 2000;83:e6)
\end{abstract}

Keywords: atrioventricular block; cystic tumour; excision

There are a variety of lesions that can arise from the right atrium and the interatrial septum and involve the conduction system. One of them is the cystic tumour of the atrioventricular (AV) nodal region, also known as mesothelioma of the AV node. ${ }^{1}$

They have been termed both "lymphangioma" and "mesothelioma of the atrioventricular node". However, the immunocytochemical features in this case and in two recently reported necropsy cases indicates an epithelial pattern of differentiation. ${ }^{2}$ This is supported by the presence of squamous epithelium and sebaceous gland-like elements. These tumours are rare and although they have been described in the literature, most of them are postmortem findings. Balasundaram and colleagues reported the first case of successful follow up after excision of a mesothelioma of the A-V node. ${ }^{3}$ Some cases have been incidentally diagnosed at necropsy, suggesting that they can remain clinically silent. ${ }^{4}$

Histopathology and

Molecular Pathology,

The General

Infirmary, Great

George Street, Leeds

LS1 3EX, UK

L A Davidson

Correspondence to: Dr Paniagua

Accepted 6 January 2000 Case report

This 59 year old woman presented with palpitations. A resting ECG and 24 hour Holter monitoring revealed sinus rhythm and various degrees of heart block. Echocardiography and MRI revealed a right atrial cystic lesion with a broad connection with the interatrial septum (fig 1). She had had a stroke 12 years previously. At

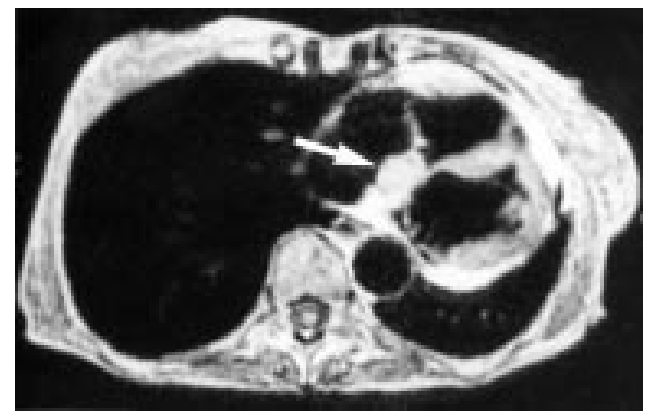

Figure 1 Magnetic resonance imaging of the thorax showing the tumour arising from the right side of the interatrial septum.

that time she was found to be in atrial fibrillation and was anticoagulated with warfarin.

The patient underwent surgery under cardiopulmonary bypass. Biatrial transeptal approach revealed a cystic mass, $3 \mathrm{~cm}$ in its greatest dimension, lying within the interatrial septum. The wall of the coronary sinus formed the lateral wall. The cyst was incised and deroofed from the right atrial surface. Approximately 3-5 ml of dark green semisolid material was removed from the cyst, which was partially excised.

Postoperatively she developed atrial flutter and various degrees of $\mathrm{AV}$ block with a slow ventricular rate that required the insertion of a permanent pacemaker. Thereafter the recovery was uneventful.

\section{Histology}

The cyst wall was composed of fibrous connective tissue showing foci of chronic inflammation and covered by a single layer of cuboidal epithelioid cells. Within the fibrous tissue were smaller cysts lined by similar cuboidal cells and containing hyaline eosinophilic material (fig 2). Focally, the cyst lining cells were stratified.

Elsewhere, there were solid nests of epithe- compact dark cells on the periphery and larger pale cells in the centre (table 1).

\section{Discussion}

The differential diagnoses of cystic epithelioid tumours in this region includes bronchogenic cysts, ectopic thyroid (struma cordis), terat- 


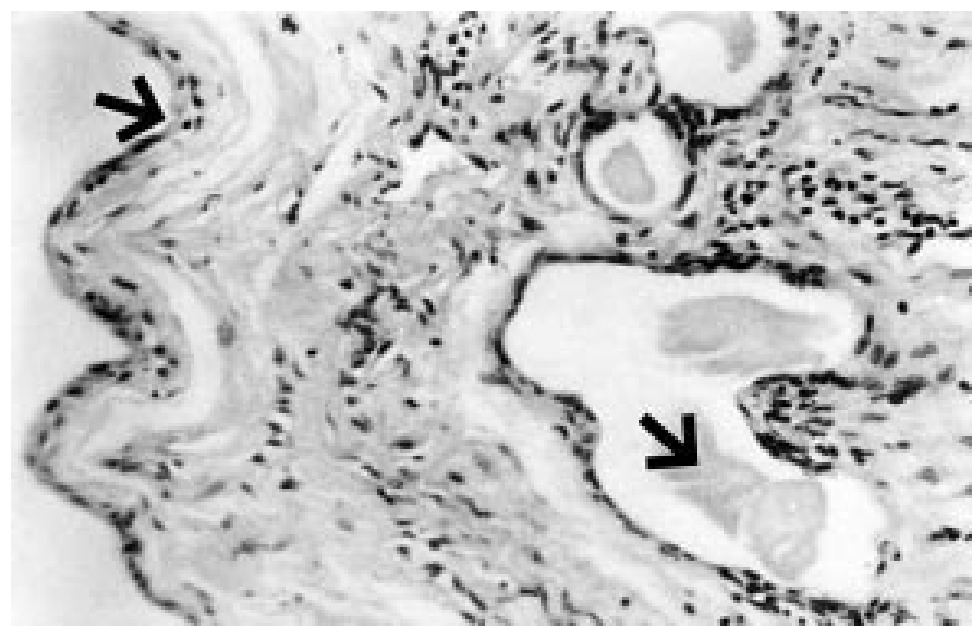

Figure 2 Photomicrograph of the cyst wall showing the large cyst lining of simple cuboidal epithelium (arrow) and within the fibrous wall of the large cyst, smaller cysts lined by similar simple cuboidal epithelium, and containing hyaline eosinophilic material (arrow).

Table 1 Antibodies used in immunocytochemical staining carried out on 5 um sections of formalin fixed paraffin embedded tissue using an avidin biotin peroxidase system with diaminobenzidine as the chromogen

\begin{tabular}{llll}
\hline Antibody & Source & Specificity & Reaction \\
\hline Antifactor VIII related antigen & Dako & Endothelium & Negative \\
HBME-1 & Dako & Mesothelium & Negative \\
CAM5.2 & Becton-Dickinson & Epithelium \& mesothelium & Positive \\
Anticarcinoembryogenic antigen & Euro Path & Epithelium & Positive \\
AUA1 & Oxoid & Epithelial glycoprotein & Positive \\
Ber EP4 & Dako & Epithelial glycoprotein & Positive \\
Antithyroglobulin & Dako & Thyroid & Negative \\
\hline
\end{tabular}

omas, metastatic adenocarcinomas and a specific pathological entity now termed "cystic tumours of the atrioventricular nodal region".

The lesion appeared histologically benign with no features to suggest that it could be a metastatic carcinoma. The histological features fit those of a cystic tumour of the atrioventricular nodal region. These are typically multi- cystic, devoid of smooth muscle, and have histologically benign epithelium showing various patterns of differentiation including the simple squamous, stratified squamous, and sebaceous gland-like patterns seen in this case. ${ }^{1}$

To our knowledge, this is the second case of cystic tumour of the AV node region that has been diagnosed antemortem and successfully treated. ${ }^{3}$ Allen and colleagues, in their study of 17 tumours in necropsy specimens, described a variety of clinical presentations including complete heart block, atrioventricular block and sudden death. ${ }^{25}$ Age of presentation ranged from newborn to 86 years. The possibility of familial occurrence of the condition has been discussed by Travers. ${ }^{4}$

In order to make the diagnosis antemortem, special mention should be paid to those patients with ECG evidence of heart block with narrow QRS complexes (limited to the AV node). ${ }^{6}$ In some cases of patients with heart block caused by this type of tumour (diagnosed postmortem), pacemaker implantation did not prevent death. ${ }^{7}$

1 Burke A, Virmani R. Tumors of the heart and great vessels. Atlas of tumor pathology. 3rd series, fascicle 16. 1995:11219.

2 Burke AP, Anderson PG, Virmani R, et al. Tumor of the atrioventricular nodal region. Arch Pathol Lab Med 1990;114:1057-62.

3 Balasundaram S, Al Halees S, Duran C. Mesothelioma of the atrioventricular node: first successful follow-up after excision. Eur Heart f 1992;13 718-19.

4 Travers H. Congenital polycystic tumor of the atrioventricular node: possible familial occurrence and critical review of reported cases with special emphasis on histogenesis. Hum Pathol 1982;13:25-35.

5 Lewman LV, Demany MA, Zimmerman HA. Congenital tumor of atrioventricular node with complete heart block and sudden death. Am $\mathcal{F}$ Cardiol 1972;29:554-7.

6 Evans DW, Stovin PGI. Fatal heart block due to 1986;56:572-4.

7 Nishida K, Kamijima G. Mesothelioma of the atrioventricular node. Br Heart $\mathcal{F}$ 1985;53:468-70. 